\title{
The Early Stage of Basal Slip in Zinc Single Crystals*
}

\author{
By Nobuo Mikuriya**, Hironobu Nishikawa**, Takao Takasaki***, \\ Hiroyuki Yokoyama**** and Masato Okochi*****
}

\begin{abstract}
An experimental study was carried out in order to investigate the contribution of basal and nonbasal dislocations (forest dislocations) to the early stage of the basal slip in $99.999 \%$ pure zinc single crystals compressed at room temperature. The relation between the basal shear stress and the shear strain was plotted and the increase in dislocation density during deformation was observed by means of the etch pit technique. The initial dislocation etch pit densities of the specimens used were $2.9 \times 10^{4} \sim 3.7 \times 10^{4} / \mathrm{cm}^{2}$ on the (0001) surface and $6.1 \times 10^{4} \sim 5.2 \times 10^{5} / \mathrm{cm}^{2}$ on the (1120) surface. From these data, the length of basal and nonbasal dislocations per unit volume was calculated using equations developed by G. Schoeck. It was assumed that the density of forest dislocations did not change during the deformation since the critical resolved shear stresses for nonbasal slip systems were considerably higher than the stresses employed in the experiments. The results obtained were as follows. (1) The multiplication stresses were approximately equal to the stresses required to break up the attractive junctions between basal and forest dislocations. (2) The yield stresses were represented by the sum of the stress necessary for the forest cutting and the internal stress due to the basal dislocations. From these results, it was concluded that the yield stress was determined by the forest cutting under the internal stress field of the basal dislocations.
\end{abstract}

(Received March 7, 1977)

\section{Introduction}

With respect to the slip deformation in zinc single crystals, some experimental investigations have been carried out on the interaction between the basal dislocations having the Burgers vectors $(a / 3)\langle 11 \overline{2} 0\rangle$ on (0001) planes and the nonbasal dislocations on the planes intersecting with (0001) planes. Stofel and Wood ${ }^{(1)}$ showed that the yield stress and flow stress in basal slip deformation of zinc single crystals increased markedly by nonbasal dislocations introduced to the specimens by the tension parallel to the hexagonal

* This paper was originally published in Japanese in J. Japan Inst. Metals, 40 (1976), 657.

** Department of Physics, Nagoya Institute of Technology, Nagoya 466, Japan.

*** Nagoya Institute of Technology, Nagoya. Present address: Research and Development Center, Tateishi Electric Co., Ltd., Nagaokakyo 617, Japan.

**** Nagoya Institute of Technology, Nagoya. Present address: Toyota Moter Co., Ltd., Toyota 471, Japan.

***** Department of Physics, Faculty Science and Engineering, Meijo University, Nagoya 468, Japan. axis. They also showed that the strength in the direction of hexagonal axis increased in specimens subjected to basal shear strain. Adams et al. ${ }^{(2)}$ examined the relation between the basal shear stress and the shear strain in single crystals of zinc containing a small quantity of aluminium, and found that with increasing impurity addition, the stress-strain curves were shifted to higher stress levels. From the dislocation density observation by the etch pit technique, they found that the addition of aluminium increased the density of nonbasal dislocations in single crystals. They explained the increase in flow stress in terms of forest dislocations resisting the motion of basal dislocations. By applying the stress pulse, Nagata et al. ${ }^{(3)}$ measured the mobility of basal dislocations in zinc single crystals whose nonbasal dislocation densities determined by $\mathrm{X}$-ray technique ranged from $10^{2}$ to $10^{6} / \mathrm{cm}^{2}$. They observed a proportional relation between the critical shear stress for the motion of basal edge dislocations and the square root of nonbasal dislocation density.

Although the results of these experiments showed a strong interaction between basal and nonbasal dislocations in zinc single crystals, it cannot be deduced that the stress 
required for basal slip deformation is exclusively determined by the nonbasal dislocation density. It goes without saying that the motion of basal dislocations is influenced by the long-range internal stresses of parallel basal glide dislocations and by the stresses required to overcome attractive and repulsive junctions with nonbasal forest dislocations. The relative importance of basal and nonbasal dislocations to the flow stress for the basal slip deformation should be decided by densities, distribution and mobility of them. So their contribution to the flow stress varies with the initial conditions of specimens and the stage of deformation. This report presents the results of experiments which were undertaken in order to obtain information about the contribution of basal and nonbasal dislocations (forest dislocations) to the early stage of basal slip deformation in zinc single crystals. The basal shear stress-shear strain behaviour and the changes in dislocation etch pit densities on $(11 \overline{2} 0)$ surfaces were measured at room temperature for specimens whose etch pit densities had been counted before testing, and the multiplication stresses of basal dislocations and the yield stresses of basal slip deformation were determined from these data. Contribution of basal and forest dislocations to these stresses is discussed in terms of the elastic interaction between dislocations.

\section{Experimental}

Single crystals were grown at a rate of $6 \mathrm{~mm} /$ h from $99.999 \%$ purity zinc under a nitrogen atmosphere by a modified Bridgman technique with a seed crystal in a high purity graphite mould. The growth direction was inclined at $40 \sim 45^{\circ}$ to the [0001] to [1100] direction. As-grown single crystals were parallelepiped with two $(1 \overline{1} \overline{2} 0)$ surfaces and were about $13 \times 10 \times 150 \mathrm{~mm}$ in dimension. A piece about $25 \mathrm{~mm}$ in length was cut from the middle part of an as-grown crystal with an acid saw along a plane vertical to the growth direction. This piece was divided to a pair of specimens in a similar manner. The pair of specimens thus obtained was formed in equal size and shape and then polished on a glass plate with a chemi- cal polishing solution ${ }^{(4)}$ infiltrated in thick gauze. After polishing, they were annealed for about $24 \mathrm{~h}$ at $350^{\circ} \mathrm{C}$ in nitrogen atmosphere; the one was used for the strain measurement and the other for the observation of dislocation etch pits. Each specimen was about $8 \times 8 \times 10$ $\mathrm{mm}$ in dimension prior to testing. Several pairs of specimens were cut from an as-grown single crystal. The crystallographic orientation and the load axis of test specimens are shown in Fig. 1.

Stress-strain measurement: As shown in Fig. 1, strain gauges were stuck on the surfaces of both sides of a specimen for strain measurement. The strain of specimen subjected to compressive load for $20 \mathrm{~s}$ was recorded by a static strainmeter having a sensitivity of $1 \times 10^{-6}$. The shear stress to strain relation was deduced from these data. The time interval between the two successive loadings was taken to be nearly equal for all specimens for the strain measurement and etch pits observation.

Observation of etch pits: The specimens for the etch pits observation were subjected to the compressive load for $20 \mathrm{~s}$. After removing the load, dislocation etch pits were made on $(11 \overline{2} 0)$ surfaces $^{(5)}$. These pits were observed under an optical microscope and an area of $4 \sim 5 \mathrm{~mm}^{2}$ on $(11 \overline{2} 0)$ surface was photographed. Then the specimen was chemically polished and stressed again. A small piece was cut from the part of the original single crystal contiguous to

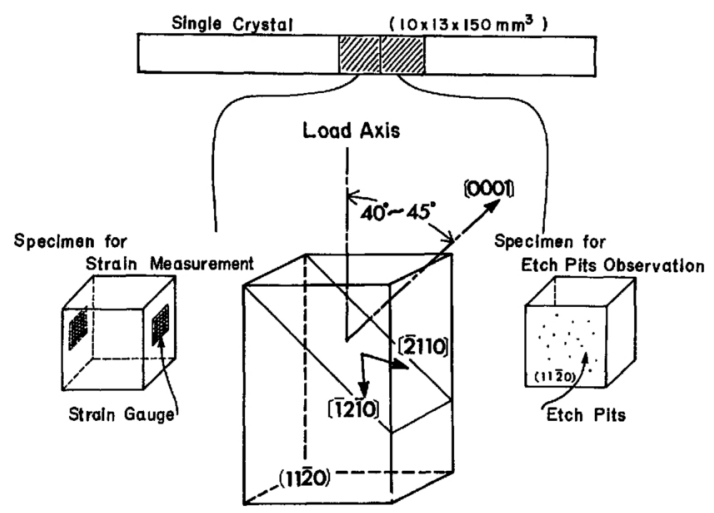

Fig. 1 Crystallographic orientation and geometry of test specimens. Two neighboring pieces were cut from a single crystal. The one was used for the observation of dislocation etch pits and the other for the strain measurement. 
Table 1 Dislocation densities of specimens before testing.

\begin{tabular}{|c|c|c|c|c|}
\hline \multirow{2}{*}{ Specimen } & \multicolumn{2}{|c|}{$\begin{array}{l}\text { Etch pit density observed on } \\
(0001) \text { and }(11 \overline{2} 0) .\left(\mathrm{cm}^{-2}\right)\end{array}$} & \multirow{2}{*}{$\begin{array}{l}\text { Basal dislocation etch } \\
\text { pit density on }(11 \overline{2} 0) \\
\qquad N_{o b}\left(\mathrm{~cm}^{-2}\right)\end{array}$} & \multirow{2}{*}{$\begin{array}{l}\text { Subboundary length } \\
\text { observed on }(11 \overline{20}) . \\
\left(\mathrm{cm}^{-1}\right)\end{array}$} \\
\hline & $N_{(0001)}$ & $N_{(11 \overline{2} 0)}$ & & \\
\hline$\diamond 13-1$ & $3.5 \times 10^{4}$ & $8.1 \times 10^{4}$ & $5.5 \times 10^{4}$ & $1.0 \times 10$ \\
\hline O 19-1 & $3.7 \times 10^{4}$ & $2.8 \times 10^{5}$ & $2.5 \times 10^{5}$ & $1.9 \times 10$ \\
\hline 19-3 & $3.7 \times 10^{4}$ & $5.2 \times 10^{5}$ & $4.9 \times 10^{5}$ & $3.3 \times 10$ \\
\hline$\square 20-3$ & $2.9 \times 10^{4}$ & $9.1 \times 10^{4}$ & $6.9 \times 10^{4}$ & $1.2 \times 10$ \\
\hline$\triangle 22-1$ & $3.4 \times 10^{4}$ & $8.3 \times 10^{4}$ & $5.7 \times 10^{4}$ & $1.3 \times 10$ \\
\hline$\Delta 22-2$ & $3.4 \times 10^{4}$ & $6.1 \times 10^{4}$ & $3.5 \times 10^{4}$ & 3.0 \\
\hline$\times 24-1$ & $3.0 \times 10^{4}$ & $9.0 \times 10^{4}$ & $6.7 \times 10^{4}$ & 7.7 \\
\hline
\end{tabular}

the specimens. The cleavage of this piece in liquid nitrogen revealed the (0001) surface, and etch pits were developed on this surface to determine the nonbasal dislocation density before testing ${ }^{(6)}$.

\section{Results}

\section{Initial dislocation densities of test specimens}

Dislocation densities on seven specimens before testing are summarised in Table 1 . The numbering of specimens in the first column is made as follows: the first serial number denotes the number of the as-grown single crystals, and the second serial number denotes the number of slices of the crystal. The symbol marked on each specimen will be used hereafter without any note. $N_{(0001)}$ in the second column is the density of etch pits which were observed on the (0001) surface and $N_{(11 \overline{2} 0)}$ in the third column is that on the $(11 \overline{2} 0)$ surface. Assuming a one-to-one correspondence between etch pits and dislocations, $N_{(0001)}$ is the density of the intersecting points of nonbasal dislocations and the (0001) plane, and $N_{(11 \overline{2} 0)}$ is the density of points where basal and nonbasal dislocations intersect with the

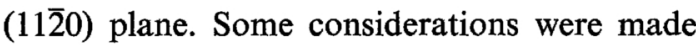
on the mechanism by which the dislocations were introduced to a single crystal grown from the melt $t^{(7)}$. It is difficult, however, to know the plane on which the dislocations lie, and is impossible to know the distribution of dislocations on each plane by the etch pit technique. In addition, it should be considered that some dislocations may be introduced in the process of preparation of test specimens. In this work that the dislocations existing in specimens before testing is assumed to lie on the planes on which their activity was experimentally observed in zinc single crystals, i.e., planes (0001), $\{10 \overline{1} 0\},\{10 \overline{1} 1\}$ and $\{11 \overline{2} 2\}^{(8)}$. So $N_{(11 \overline{2} 0)}$ is the etch pit density due to the dislocations in the planes of these four sets and $N_{(0001)}$ is that in the planes $\{10 \overline{1} 0\},\{10 \overline{1} 1\}$ and $\{11 \overline{2} 2\}$. If the fraction of etch pits formed on the (0001) surface by the dislocations lying on $\{10 \overline{1} 0\},\{10 \overline{1} 1\}$ and $\{11 \overline{2} 2\}$ planes are $p_{1}$, $p_{2}$ and $p_{3}$ (here $p_{1}+p_{2}+p_{3}=1$ ), the densities of etch pits representing these dislocations are $p_{1} N_{(0001)}, p_{2} N_{(0001)}$ and $p_{3} N_{(0001)}$, respectively. Further, it is assumed that the dislocations on three $\{10 \overline{1} 0\}$, six $\{10 \overline{1} 1\}$ and $\{11 \overline{2} 2\}$ planes are distributed homogeneously and in the same densities. Accordingly, the method developed by Schoeck ${ }^{(9)}$ can be employed to calculate the length $L$ per unit volume of the dislocation line existing on an arbitrary plane from the etch pit density $N$ per unit area on an arbitrary observed surface. The numerical calculation gave $L_{\{10 \overline{1} 0\}}=1.6 p_{1} N_{(0001)}, L_{\{10 \overline{1} 1\}}=$ $1.7 p_{2} N_{(0001)}$ and $L_{\{11 \overline{2} 2\}}=1.8 p_{3} N_{(0001)}$. The dislocation etch pit densities observed on the

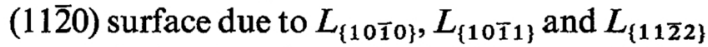
were obtained to be $N^{\prime}=0.42 L_{\{10 \overline{1} 0\}}=0.42 \times$ $1.6 p_{1} N_{(0001)}, \quad N^{\prime \prime}=0.48 L_{\{10 \overline{1} 1\}}=0.48 \times 1.7 p_{2}$ $N_{(0001)} \quad$ and $\quad N^{\prime \prime \prime}=0.44 L_{\{1122\}}=0.44 \times 1.8 p_{3}$ $N_{(0001)}$. The etch pit density, on the $(11 \overline{2} 0)$ surface, induced by nonbasal dislocations is $N^{\prime}+N^{\prime \prime}+N^{\prime \prime \prime} \approx 0.76\left(p_{1}+p_{2}+p_{3}\right) N_{(0001)}=$ $0.76 N_{(0001)}$, and therefore the one induced by basal dislocations is $N_{\mathrm{ob}}=N_{(11 \overline{2} 0)}-0.76 N_{(0001)}$. 
The values of $N_{\mathrm{ob}}$ listed in Table 1, which are the densities of etch pits corresponding to basal dislocations observed on the (1120) surface before testing, were calculated using such a method. In this table, the subboundary length observed on the $(11 \overline{2} 0)$ surface for each specimen is the total length of pits arrays in which the individual pits cannot be distinguished from each other.

The density of etch pits on the (0001) surface could not be observed during and after the testing; that is, the change of nonbasal dislocations induced by basal slip could not be known. However, it is believed that this change is small. The reason is as follows. It has been reported that the dislocations of $\langle 11 \overline{2} 0\rangle\{10 \overline{1} 0\}$ slip system are active only in the temperature range of $250 \sim 400^{\circ} \mathrm{C}$ and are not operative at room temperature ${ }^{(10)}$. The operation of the $\langle 11 \overline{2} 3\rangle\{11 \overline{2} 2\}$ slip system, on the other hand, has been observed at room temperature ${ }^{(11) \sim(13)}$. There are no detailed data on the multiplication of dislocations on this slip system. However, when the specimen prepared from the same material and grown by the same method as that used in this experiment was subjected to compressive static load along the [0001] axis for $20 \mathrm{~s}$, it yielded at a resolved shear stress of about $150 \mathrm{~g} / \mathrm{mm}^{2}$. Although the increase in etch pit density on the (0001) surface from the initial value of $2.1 \times 10^{4}$ to $1.4 \times 10^{5} / \mathrm{cm}^{2}$ was observed immediately after the yielding, the change in etch pit density could not be observed prior to yielding ${ }^{(14)}$. A similar experiment has been done using the specimen with an initial etch pit density of $4.2 \times 10^{3} / \mathrm{cm}^{2}$ on the (0001) surface. The etch pit density of this specimen on the (0001) surface increased to $5.9 \times 10^{3} / \mathrm{cm}^{2}$ at a resolved shear stress of $110 \mathrm{~g} / \mathrm{mm}^{2}$ and this specimen yielded at $140 \mathrm{~g} / \mathrm{mm}^{2}$. It was conceivable from the arrangement of etch pits on the observation surfaces (0001) and (1010) that the increase in etch pit density resulted from the multiplication of dislocations in the $\langle 11 \overline{2} 3\rangle\{11 \overline{2} 2\}$ slip system. Blish et al. ${ }^{(13)}$ measured the dislocation velocity of this slip system from the growth velocity of the slip band. According to their report, the motion of edge dislocations could not be observed if the resolved shear stress was less than about $80 \mathrm{~g} / \mathrm{mm}^{2}$ at room temperature. In this report, the maximum value of resolved shear stress applied to the $\langle 11 \overline{2} 3\rangle$ $\{11 \overline{2} 2\}$ slip system was about $60 \%$ of the value for the $\langle 11 \overline{2} 0\rangle(0001)$ basal slip system. So the maximum value of resolved shear stress applied to the $\langle 11 \overline{2} 3\rangle\{11 \overline{2} 2\}$ slip system was about $40 \mathrm{~g} / \mathrm{mm}^{2}$ for specimen $19-3$, and less for other specimens. Accordingly, the pronounced motion of dislocations which may change their density seems not to have happened in the specimens used. From electron microscope observations, Price ${ }^{(15)}$ confirmed $\{10 \overline{1} 1\}$ planes as the cross glide planes of screw dislocations with $(1 / 3)<11 \overline{2} 3\rangle$ Burgers vector.

On the other hand, it was reported that a few dislocations moved under the stress below $1 \mathrm{~g} / \mathrm{mm}^{2}$ in copper single crystals ${ }^{(16)}$. Therefore we cannot draw the conclusion that the density of nonbasal dislocations did not change during this experiment. In this report the arguments will be put forward on the assumption that the density of nonbasal dislocations does not change.

\section{Stress and strain}

The relation between the logarithm of resolved shear stress $\tau$ and that of shear strain $\gamma$ in the slip system $\langle 11 \overline{2} 0\rangle(0001)$ is shown in Fig. 2. In general, when the relation between $\tau$ and $\gamma$ is expressed as $\tau=k \gamma^{n}$ ( $k$ is a constant and $n>0)$, the yield point can be determined as the point where the value of $n$ is minimum. The yield stress for each specimen is defined

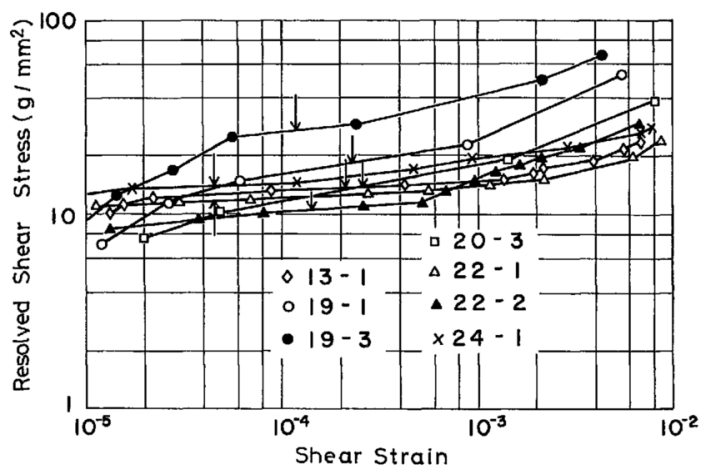

Fig. 2 Relation between resolved shear stress and shear strain in the slip system $\langle 11 \overline{2} 0\rangle(0001)$. The arrows indicate the yield point for each specimen. 
as the stress at the middle point in the portion of the minimum slope of the curve describing the stress-strain relation in Fig. 2. The arrows indicate the yield point and the values of yield stress were $\mathbf{A}: 10.5 \mathrm{~g} / \mathrm{mm}^{2}, \triangle: 11.5 \mathrm{~g} / \mathrm{mm}^{2}$, $\diamond: 13.0 \mathrm{~g} / \mathrm{mm}^{2}, \quad \times: 13.5 \mathrm{~g} / \mathrm{mm}^{2}, \quad \square: 14.0$ $\mathrm{g} / \mathrm{mm}^{2}, \bigcirc: 18.5 \mathrm{~g} / \mathrm{mm}^{2}$ and $0: 25.8 \mathrm{~g} / \mathrm{mm}^{2}$. Even if specimens were cut from the same mother crystal, the values of yield stress differed from each other. This fact suggests that the yield stress was affected by the initial basal dislocation density $N_{\mathrm{ob}}$.

\section{Stress and basal dislocation density}

Photograph 1(a) (f) show the changes in density and arrangement of dislocations of specimen $\bigcirc 19-1$ at several different levels of the shear stresses, which were taken at the same area on the $(11 \overline{2} 0)$ surface. There occurred little changes in the etch pit density and its distribution with an application of a shear stress $7 \mathrm{~g} / \mathrm{mm}^{2}$ [Photo. 1(b)]. After the application of shear stress $14 \mathrm{~g} / \mathrm{mm}^{2}$, the etch pit density has somewhat increased. There were pit rows on slip traces although the traces were scarce, and it can be seen that the subboundaries are partly broken [Photo. 1(c)].
Because the yield stress of this specimen was $18.5 \mathrm{~g} / \mathrm{mm}^{2}$, Photo. 1(d), (e) and (f) show the feature of the deformed specimen after yielding. In Photo. 1(d), the slip traces become closer in distance, the dislocation etch pits are dense in the slip traces, and hindrance of dislocation motion by the subboundaries can be observed. In Photo. 1(e), there are many slip traces uniformly distributed over the whole surface.

The increase in etch pit density on the (1120) surface was observed for all the specimens listed in Table 1. Because it is assumed that there is no change in the nonbasal dislocation density, the basal dislocation should be responsible for the increase of the etch pit density. The plots of basal dislocation density $N_{b}$ vs resolved shear stress $\tau$ are shown in Fig. 3. Figure 3(a) shows the plots for four specimens having the different initial basal dislocation densities $N_{\mathrm{ob}}$. In this figure, the specimens $\bigcirc 19-1$ and 19-3 were cut from a crystal, and $\Delta 22-1$ and $\Delta$ 22-2 from another crystal. Figure 3(b) shows the plots for three specimens having a similar value of $N_{\mathrm{ob}}$ and cut from the different mother crystals. The data for the specimens $\square 20-3$ and $\times 24-1$ having a very similar value of $N_{\mathrm{ob}}$ fall on a
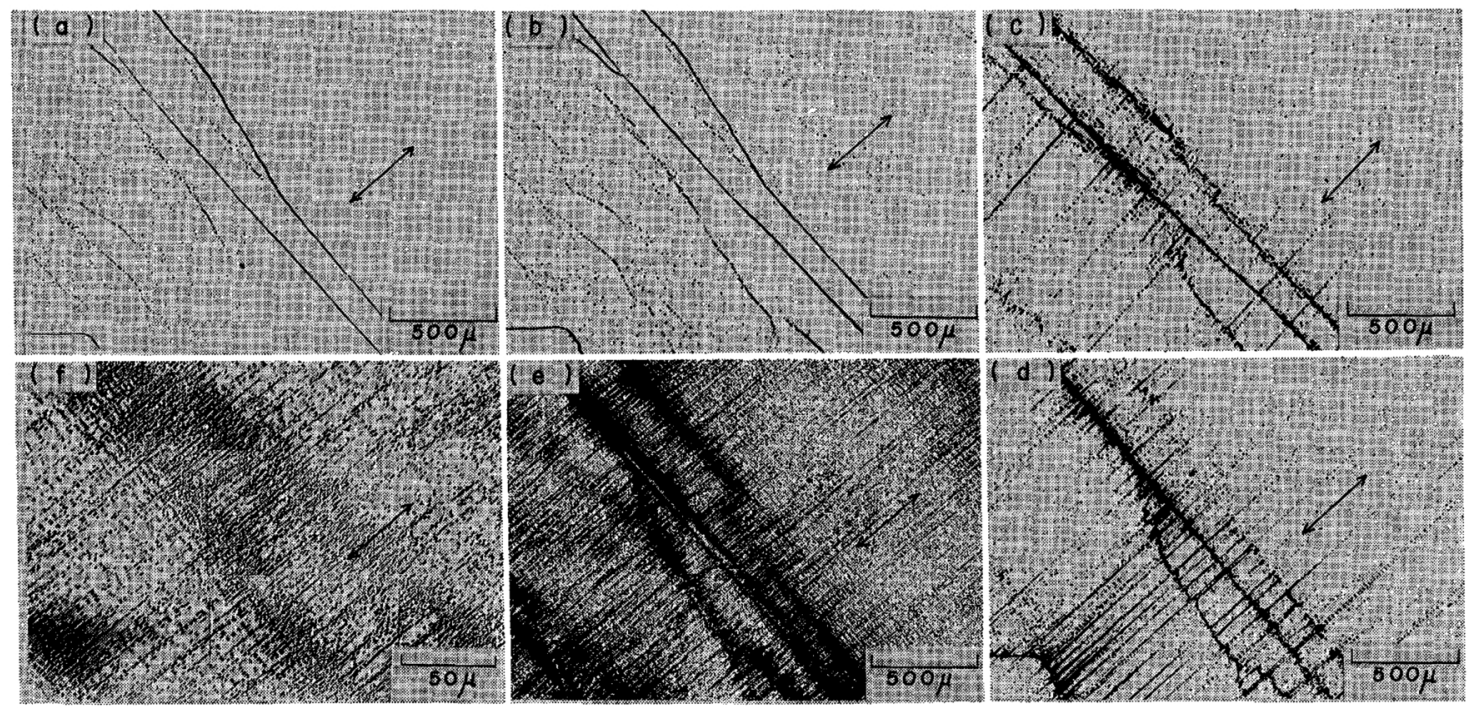

Photo. 1 Micrographs showing the basal dislocation density changes resulting from basal shear stress. Each photograph shows the same area on the (1120) surface of specimen 19-1. Basal glide plane trace is parallel to the arrow. (a) before testing, (b) $7 \mathrm{~g} / \mathrm{mm}^{2}$ shear stress, (c) $14 \mathrm{~g} / \mathrm{mm}^{2}$ shear stress, (d) $28 \mathrm{~g} / \mathrm{mm}^{2}$ shear stress, (e) $54 \mathrm{~g} / \mathrm{mm}^{2}$ shear stress, and (f) enlarged photograph of (e). Yield stress of this specimen is $18.5 \mathrm{~g} / \mathrm{mm}^{2}$. 


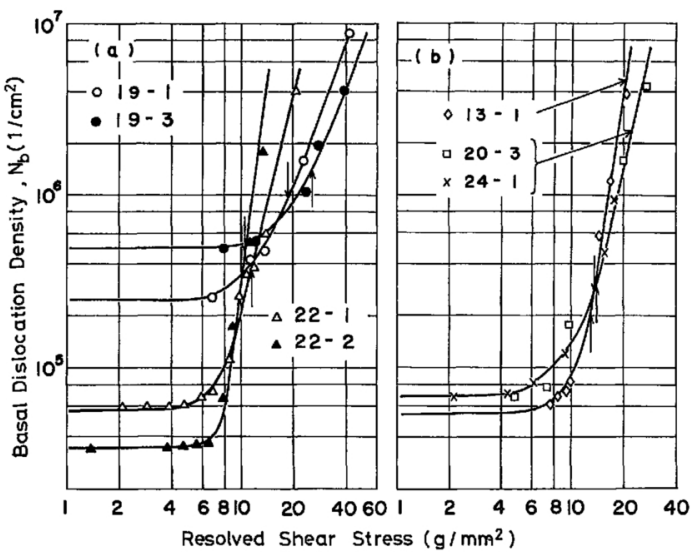

Fig. 3 Plots of basal dislocation density versus resolved shear stress. (a) for specimens 19-1, 19-3, 22-1 and 22-2, (b) for specimens 13-1, 20-3 and 24-1. The vertical arrows indicate the yield point for each specimen.

smooth curve. From the curves in Fig. 3, the stress necessary for dislocation multiplication can be estimated. The multiplication stresses for seven specimens listed in Table 1 are in the range of $7 \sim 12 \mathrm{~g} / \mathrm{mm}^{2}$, and it can be seen that the specimens having the large values of $N_{\mathrm{ob}}$ tend to require the large multiplication stress. It is also shown that after yielding, the relation between dislocation density and shear stress for each specimen is still influenced by the initial dislocation density.

\section{Discussion}

\section{Multiplication stress}

The nonbasal dislocations existing in specimens give the resistance force as forest dislocations for the motion of basal dislocations. The calculations of stress required to overcome the attractive and repulsive junctions formed by the intersection of both types of dislocations have been made by Saada ${ }^{(17)}$ in $f c c$ crystal structures. The result is given as $\tau=G b / K h_{f}$. In this equation, $G$ is the shear modulus, $b$ the magnitude of Burgers vector of dislocations which is assumed to be the same for both dislocations, $h_{f}$ the average distance between attractive or repulsive junctions, and $K$ a constant whose value is 2.5 for attractive junctions and 10 for repulsive junctions. The
Table 2 Attractive reactions between basal and nonbasal dislocations in zinc crystals.

\begin{tabular}{|c|c|c|}
\hline$b_{1}(0001)$ & $b_{2}\{10 \overline{10}\}$ & $b_{3}(0001)$ \\
\hline \multicolumn{3}{|c|}{$\begin{aligned} \frac{1}{3}[11 \overline{2} 0]+\frac{1}{3}[1 \overline{2} 10](10 \overline{1} 0) & =\frac{1}{3}[2 \overline{1} \overline{1} 0] \\
+\frac{1}{3}[\overline{2} 110](0 \overline{1} 10) & =\frac{1}{3}[\overline{1} 2 \overline{1} 0]\end{aligned}$} \\
\hline$b_{1}(0001)$ & $b_{2}\{10 \overline{1} 1\}$ & $b_{3}(0001)$ \\
\hline \multicolumn{3}{|c|}{$\begin{aligned} \frac{1}{3}[11 \overline{2} 0]+\frac{1}{3}[1 \overline{2} 10](10 \overline{1} 1) & =\frac{1}{3}[2 \overline{1} \overline{1} 0] \\
+\frac{1}{3}[1 \overline{2} 10](10 \overline{1} \overline{1}) & =\frac{1}{3}[2 \overline{1} 10] \\
+\frac{1}{3}[\overline{2} 110](01 \overline{1} 1) & =\frac{1}{3}[\overline{1} 2 \overline{1} 0] \\
+\frac{1}{3}[\overline{2} 110](01 \overline{1} \overline{1}) & =\frac{1}{3}[\overline{1} 2 \overline{1} 0]\end{aligned}$} \\
\hline$b_{1}(0001)$ & $b_{2}\{11 \overline{2} 2\}$ & $b_{3}\{11 \overline{2} 2\}$ \\
\hline \multicolumn{3}{|c|}{$\begin{aligned} \frac{1}{3}[11 \overline{2} 0]+\frac{1}{3}[1 \overline{2} 13](\overline{1} 2 \overline{1} 2) & =\frac{1}{3}[\overline{2} 113](2 \overline{1} 12) \\
+\frac{1}{3}[1 \overline{2} 1 \overline{3}](1 \overline{2} 12) & =\frac{1}{3}[\overline{2} 11 \overline{3}](\overline{2} 112) \\
+\frac{1}{3}[\overline{2} 113](2 \overline{1} 12) & =\frac{1}{3}[1 \overline{1} 13](\overline{1} 2 \overline{1} 2) \\
+\frac{1}{3}[\overline{2} 11 \overline{3}](\overline{2} 112) & =\frac{1}{3}[1 \overline{2} 1 \overline{3}](1 \overline{2} 12)\end{aligned}$} \\
\hline$b_{1}(0001)$ & $b_{2}\{11 \overline{2} 2\}$ & $b_{3}\{10 \overline{1} 0\}$ \\
\hline$\frac{1}{3}[11 \overline{2} 0]$ & $\begin{array}{l}\frac{1}{3}[\overline{1} \overline{1} 23](11 \overline{2} 2 \\
\frac{1}{3}[\overline{1} 12 \overline{3}](\overline{1} \overline{1} 22\end{array}$ & $\begin{array}{l}=[0001] \\
=[000 \overline{1}]\end{array}$ \\
\hline
\end{tabular}

value of $K$ suggests that the breaking stress of the attractive junction is larger than that of the repulsive junction. Detailed calculation has not been made for $h c p$ structures, so Saada's equation for $f c c$ structures is used in the present investigation. The dislocation reactions which are most likely to form the attractive junctions in zinc are the reactions between basal dislocations with Burgers vector $(a / 3)\langle 11 \overline{2} 0\rangle$ and forest dislocations in $\langle 11 \overline{2} 0\rangle$ $\{10 \overline{1} 0\},\langle 11 \overline{2} 0\rangle\{10 \overline{1} 1\}$ and $\langle 11 \overline{2} 3\rangle\{11 \overline{2} 2\}$ slip systems. The number of these reactions is then twelve as shown in Table 2 . In Table $2, b_{1}$ is the Burgers vector of basal dislocations, $\boldsymbol{b}_{2}$ that of forest dislocations which interact with basal ones, and $b_{3}$ that of junction dislocations. The crystal planes in which these dislocations exist are also included in this table. Of six dislocations in the $\langle 11 \overline{2} 0\rangle\{10 \overline{1} 0\}$ slip system, two dislocations form the attractive junctions. Four dislocations among the twelve dislocations in the $\langle 11 \overline{2} 0\rangle\{10 \overline{1} 1\}$ slip system and six dislocations among the twelve dislocations in the $\langle 11 \overline{2} 3\rangle\{11 \overline{2} 2\}$ slip system form attractive junctions. Consequently the forest dislocation densities forming the attractive junctions with basal dislocations are $(1 / 3) L_{\{10 \overline{1} 0\}}+(1 / 3)$ $L_{\{10 \overline{1} 1\}}+(1 / 2) L_{\{11 \overline{2} 2\}} \approx 0.67 N_{(0001)}$, and the 
mean spacings of junctions are $h_{f}=1$ / $\sqrt{0.67 N_{(0001)}}$. Using the etch pit counts in Table 1 , the values of $h_{f}$ lie between $6.4 \times$ $10^{-3}$ and $7.2 \times 10^{-3} \mathrm{~cm}$ for seven specimens.

The multiplication of basal dislocations occurs at the stress range of about $8 \sim 12$ $\mathrm{g} / \mathrm{mm}^{2}$ before yielding as can be seen in Fig. 3 . According to the multiplication mechanism proposed by Frank and Read ${ }^{(18)}$, the dislocation line of length $l$ becomes operative as the dislocation source by applying the stress $\tau=G a / l$. If the Frank-Read mechanism is responsible for the basal dislocation multiplication, the dislocation sources of $l=8.7 \times 10^{-3} \sim$ $1.3 \times 10^{-2} \mathrm{~cm}$ or greater should exist on (0001) planes prior to the yielding, with $G=$ $3.9 \times 10^{6} \mathrm{~g} / \mathrm{mm}^{2}$ and $a=2.67 \times 10^{-8} \mathrm{~cm}$ for zinc crystals. Comparison of the values of $l$ with $h_{f}$ suggests the following. In order for the dislocation segments of length $l$ to serve as Frank-Read sources, they must overcome the attractive junctions with forest dislocations which are distributed with average distance $h_{f}$. It follows that the multiplication of basal dislocations begins when the applied stress attains at least the same value as the resistance by attractive junctions. Taking the magnitude of Burgers vector $b$ as $2.67 \times 10^{-8} \mathrm{~cm}$ and the average distance $h_{f}$ between attractive junctions as $6.4 \sim 7.2 \times 10^{-3} \mathrm{~cm}$, the stresses required to overcome the attractive junctions $\tau_{f}$ are obtained to be $5.7 \sim 6.4 \mathrm{~g} / \mathrm{mm}^{2}$ from Saada's equation. These values are approximately equal to the observed multiplication stresses. It may be considered that the breaking up of the attractive junctions with forest dislocations is closely related to the multiplication of basal dislocations.

\section{Yield stress}

Young ${ }^{(19)}$ has shown that the yield stress of copper single crystals was related only to the mother crystal from which the specimen was cut. He concluded that the yield stress was governed by the impurity atoms within the crystal. In the present observation, the yield stress is not related to the mother crystal of the specimen but has a tendency to depend on the initial basal dislocation density $N_{\mathrm{ob}}$ as described in section III. The yielding, therefore, cannot

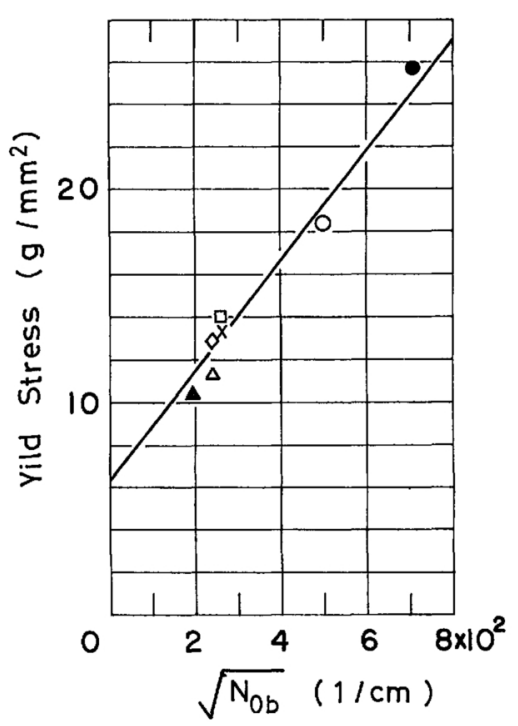

Fig. 4 Relation between the yield stress for basal slip and the square root of initial basal dislocation density.

be ascribed to the impurity atoms in specimens. The relation of yield stress $\tau_{y}$ to $\sqrt{N_{\mathrm{ob}}}$ is shown in Fig. 4 and is represented by the least-squares-fitted line:

$$
\tau_{y}=\tau_{0}+0.25 G a \sqrt{N_{\mathrm{ob}}} \quad\left(\mathrm{g} / \mathrm{mm}^{2}\right)
$$

with $\tau_{0}=6.2 \mathrm{~g} / \mathrm{mm}^{2}$. The stress $\tau_{0}$ corresponding to $N_{\mathrm{ob}}=0$ is the portion of the yield stress which is unrelated to the initial basal dislocation density. Namely, the yield stress is represented by the sum of a term unrelated to the initial basal dislocation density $N_{\mathrm{ob}}$ and a term proportional to the square root of $N_{\mathrm{ob}}$.

At the yielding, the many dislocations on the slip plane must cover a long distance as compared with the mean spacing between dislocations, and to continue the moving they must overcome the opposing stress peak. This stress is the yield stress of crystals. The condition for many dislocations to move a fairly long distance through the crystal is to overcome the attractive junctions with forest dislocations, as suggested in section IV. 1. The forest dislocation density $N_{f}\left(\approx 0.67 N_{(0001)}\right)$ forming attractive junctions with glide dislocations may be assumed to be the same for each specimen used, because its range was $1.9 \times$ 
$10^{4} / \mathrm{cm}^{2}$ to $2.5 \times 10^{4} / \mathrm{cm}^{2}$. If the constant $\tau_{0}$ is related to $N_{f}$ by $\alpha G a \sqrt{N_{f}}$, then eq. (1) is expressed as

$$
\tau_{y}=\alpha G a \sqrt{N_{f}}+\beta G a \sqrt{N_{\mathrm{ob}}} \quad\left(\mathrm{g} / \mathrm{mm}^{2}\right),
$$

where $\alpha$ and $\beta$ are constants. Using the mean value $2.3 \times 10^{4} / \mathrm{cm}^{2}$ of $N_{f}$ for seven specimens, the constant $\alpha$ is about 0.39 . Comparison of the value of $\alpha$ with that of the coefficient $1 / K$ of the stress required to break up the attractive junctions shown by Saada suggests that the first term in eq. (2) is the stress necessary for the forest cutting. The second term is the stress relating to the basal dislocations. The dislocations, lying on the parallel glide planes, having the same Burgers vector with a moving dislocation and being distributed with density $N$, give the resistance stress of $\tau=\beta G a \sqrt{N}$ for a moving dislocation, where $\beta$ is a constant of the order of magnitude of $0.1 \sim 0.2$. But its exact value may vary with the arrangement of dislocations in specimen ${ }^{(20)}$. According to the results of this test, the value of $\beta$ is 0.25 as shown in eq. (1) and is slightly larger than the value given by other authors. The reason is considered as follows. At the yield point, the basal dislocation densities considerably increase as can be seen in Fig. 3, so the internal stresses may be large compared with the calculated value of $N_{\mathrm{o} b}$. From such a consideration, the second term may be interpreted as the internal stress field due to the basal dislocations. This results predicts that the yield stress is represented by the sum of the stress required to break the attractive junctions with forest dislocations and the internal stress due to the basal dislocations. Namely, it seems to be the mechanism controlling the yielding that the moving dislocations run over a long distance by cutting forest dislocations under the internal stress field of basal dislocations.

\section{Conclusion}

The contribution of basal and nonbasal dislocations (forest dislocations) to the early stage of basal slip has been studied in zinc single crystals compressed at room temperature. The specimens used were the single crystals grown from $99.999 \%$ pure zinc by a modified Bridgman technique. The initial dislocation etch pit densities of specimens were $2.9 \sim 3.7 \times 10^{4} / \mathrm{cm}^{2}$ on the (0001) surface and $6.1 \times 10^{4} \sim 5.2 \times 10^{5} / \mathrm{cm}^{2}$ on the $(11 \overline{2} 0)$ surface. From these data, the densities of basal and nonbasal dislocations were calculated by assuming the homogeneous and uniform distribution of them. It was assumed that the forest dislocation densities did not change during the deformation. The results obtained were as follows. (1) The multiplication stresses of basal dislocations were approximately equal to the stress required to break out the attractive junctions between basal and forest dislocations. (2) The yield stresses were represented by the sum of the stress necessary for the forest cutting and the internal stress due to the basal dislocations. From these results, it was concluded that the yielding is controlled by the forest cutting under the internal stress field of the basal dislocations.

\section{REFERENCES}

(1) E. J. Stofel and D. S. Wood: Fracture of Solid, ed. by D. C. Drucker and J. J. Gilman, Interscience, New York, (1963), p. 521.

(2) K. H. Adams and T. Vreeland, Jr.: Trans. Met. Soc. AIME, 242 (1968), 132.

(3) N. Nagata and T. Vreeland, Jr.: Phil. Mag., 25 (1972), 1137.

(4) P. P. Shinha and P. A. Beck: J. Appl. Phys., 32 (1961), 1222.

(5) N. Mikuriya and M. Okochi: Japan J. Appl. Phys., 6 (1967), 1141.

(6) H. S. Rosenbaum and M. M. Saffeen: J. Appl. Phys., 32 (1961), 1866.

(7) A. Ookawa: Bull. of Amer. Phys. Soc., 13 (1968), 444.

(8) J. P. Hirth and J. Lothe: Theory of Dislocations, McGraw-Hill, (1968), p. 261.

(9) G. Schoeck: J. Appl. Phys., 33 (1962), 1745.

(10) J. J. Gilman: Trans. AIME, 206 (1956), 1326.

(11) H. S. Rosenbaum: Acta Met., 9 (1961), 742.

(12) K. H. Adams, R. C. Blish and T. Vreeland, Jr.: Mater. Sci. Eng., 2 (1967), 201.

(13) R. C. Blish and T. Vreeland, Jr.: J. Appl. Phys., 40 (1969), 884.

(14) N. Mikuriya and K. Mochinaga: Bull. Nagoya Inst. Tech., 24 (1972), 73. (in Japanese).

(15) P. B. Price: Phil. Mag., 5 (1960), 873.

(16) S. Kitajima, H. Tonda and H. Kaieda: Proc. Int. Conf. on Strength of Metals and Alloys, Supplement to Trans. JIM, Vol. 9, (1968), 740.

(17) G. Saada: Acta Met., 8 (1960), 200, 841.

(18) For example J. Friedel: Dislocations, Pergamon Press, (1964), p. 224.

(19) F. W. Young, Jr.: J. Appl. Phys., 33 (1962), 963.

(20) D. Mclean: Mechanical Properties of Metals, John Wiley and Sons, Inc., (1965), p. 104. 